\title{
Analysis of a Non-linear Partial Difference Equation, and Its Application to Cardiac Dynamics
}

\author{
MICHAEL D. STUBNA ${ }^{a, *}$, RICHARD H. RAND ${ }^{a, \dagger}$ and \\ ROBERT F. GILMOUR Jr. ${ }^{b}$ \\ a Department of Theoretical and Applied Mechanics, Cornell University, \\ 212 Kimball Hall, Ithaca, NY 14853, USA; ' Department of Physiology, \\ Cornell University, Ithaca, NY 14853, USA
}

(Received 5 January 2002; In final form 1 May 2002)

\begin{abstract}
A model of a strip of cardiac tissue consisting of a one-dimensional chain of cardiac units is derived in the form of a non-linear partial difference equation. Perturbation analysis is performed on this equation, and it is shown that regular perturbations are inadequate due to the appearance of secular terms. A singular perturbation procedure known as the method of multiple scales is shown to provide good agreement with numerical simulation except in the neighborhood of a singularity of the slow flow. The perturbation analysis is supplemented by a local numerical simulation near this singularity. The resulting analysis is shown to predict a "spatial bifurcation" phenomenon in which parts of the chain may be oscillating in period-2 motion while other parts may be oscillating in higher periodic motion or even chaotic motion.
\end{abstract}

\footnotetext{
*stubna@cornell.edu.

†Corresponding author. E-mail: rhr2@cornell.edu
} 
Keywords: Partial difference equation; Non-linear; Cardiac dynamics; Singular perturbation method; Spatial bifurcations

AMS Subject Classifications: 39A11; 92C20

\section{INTRODUCTION}

In this work, we will derive and analyze a non-linear partial difference equation which has application to cardiac dynamics. We begin with a review of the relevant biology.

The major cause of human death in the United States is catastrophic disturbances in electrical rhythms in the heart [7]. Ventricular fibrillation, a particularly lethal phenomenon, consists of the heart being totally ineffectual at pumping blood due to disorganized irregular patterns of electrical activity in the heart. The electrical patterns of excitation during fibrillation are disordered in both space and time, and exhibit a lack of synchrony which is essential for normal heart functioning.

An understanding of how normal regular electrical patterns in the heart ultimately progress to lethal phenomena such as ventricular fibrillation is at this point incomplete. Previous studies have suggested that the disordered behavior of the heart during fibrillation may arise from orderly behavior through a series of bifurcations as some parameters of the mathematical models or characteristics of the biological systems are changed. In previous works, in a step towards understanding cardiac dynamics, periodically-excited heart tissue has been modeled as a single one-dimensional iterated map $[1,3,4,8]$. In this paper, we extend these models to a chain of coupled one-dimensional maps and attempt to understand the dynamics and bifurcations of the chain. This model is still far simpler than a realistic three-dimensional model of the spatially complex heart, but it does accurately reflect some biological situations (namely the Purkinje fibers found in the heart), as well as provide an analytically tractable model of complex cardiac rhythms in one spatial dimension.

Our mathematical model takes the form of a non-linear partial difference equation. In the following section, we outline the biological motivation behind this work. In Third section, we present a derivation of the governing equation. Fourth-Six sections contain an analysis of the dynamics and 
bifurcations of the model, and involve the use of singular perturbation methods as well as numerical simulation.

\section{BIOLOGY OF THE PROBLEM}

The wave of electrical activity which propagates through the heart is the aggregate behavior of many excitable cells, each producing an action potential. An action potential for a single cell is a measurable quantity, namely the voltage difference across the membrane of the cell, which is produced either when the cell is excited above threshold (as in the case of normal heart cells) or spontaneously in a periodic nature (as in pacemaker cells). Single neuron models and single-compartment models of heart tissue have been widely studied, and the generation of action potentials in single neurons is well understood biologically and from a modeling standpoint.

Even though there are usually many ionic currents involved in the production of an action potential, it is possible in some cases to capture the dynamics of the system with particularly simple models. For example, experiments have shown that one can characterize the dynamics of a small patch of heart tissue with a single function which relates the duration of an action potential to that of the duration of the previous refractory or rest period [3]. This is based on the idea that the tissue's response to a stimulus is strongly dependent on how long that tissue has had to rest and recover from the previous action potential.

In experiments, such a system is periodically excited by an applied stimulus which results in a one-dimensional iterated map $x_{n+1}=f\left(x_{n}\right)$, with $n$ being the stimulus number, $x$ being the action potential duration corresponding to the stimulus $n$, and $f$ being derived from the experimentally determined function which relates the action potential duration to the previous rest interval. One-dimensional maps have been widely studied mathematically and are well understood. The resulting behavior, if the function $f$ is non-monotic, can include such phenomenon as period doubling and chaos as a bifurcation parameter (in this case the period of the forcing) is changed. These simple models show remarkably good agreement with biological experiments on heart tissue [8]. In the present work, we will investigate the dynamics of a coupled chain of such one-dimensional maps. 


\section{DERIVATION OF THE GOVERNING EQUATION}

In this work, we will consider a strip of cardiac tissue to be composed of a string of individual cardiac units, each of which could be loosely interpreted as a small conglomeration of synchronous excitable cells.

As shown in Fig. 1, each individual cardiac unit involves a point in time $F$ at which the unit "fires", i.e. undergoes a sudden increase in voltage, and a second point in time $Q$ at which the cell becomes "quiescent", i.e. the voltage drops to zero. Each of these units fires repetitively, so we denote by $F_{i, n}$ the time at which the $i$ th unit fires its $n$th pulse beat, and $Q_{i, n}$ as the time at which $i$ th unit becomes quiescent after the $n$th pulse beat. The duration for which the $i$ th unit has a non-zero voltage during its $n$th pulse beat is denoted by $\mathrm{APD}_{i, n}$ (for the action potential duration). The duration for which the $i$ th unit has a zero voltage during its $n$th pulse beat is denoted by $\mathrm{DI}_{i, n}$ (for the diastolic interval).

Our model is based on two experimental observations, which we take as facts. The first fact is that for a single cardiac unit, the duration of an action potential is solely determined by a single function of the previous diastolic interval. That is, in terms of our model,

$$
\mathrm{APD}_{i, n}=f\left(\mathrm{DI}_{i, n-1}\right) .
$$

The second experimental fact is that the speed of propagation of the $n$th excitation signal from the $i$ th unit to the $(i+1)$ th unit is also solely determined by a single function of the previous diastolic interval. We denote this speed as $\mathrm{CV}_{i, n}$ for conduction velocity and have therefore

$$
\mathrm{CV}_{i, n}=g\left(\mathrm{DI}_{i, n-1}\right) .
$$

Note that $\mathrm{CV}_{i, n}$ refers only to the point in the cycle where the unit becomes excited, i.e. where the voltage undergoes a sudden increase.

The governing equation for the chain can now be constructed. Each unit is assumed to receive a signal from the unit to the left of it. The first unit in the chain is being excited periodically by the experimenter, and we treat this as a boundary condition, see below. Our derivation involves relating the $n$th period of the $i$ th unit, which we represent by $P_{i, n}$, to the $n$th period of the $(i+1)$ th unit $P_{i+1, n}$. It is evident that

$$
P_{i, n}=\mathrm{APD}_{i, n}+\mathrm{DI}_{i, n} .
$$




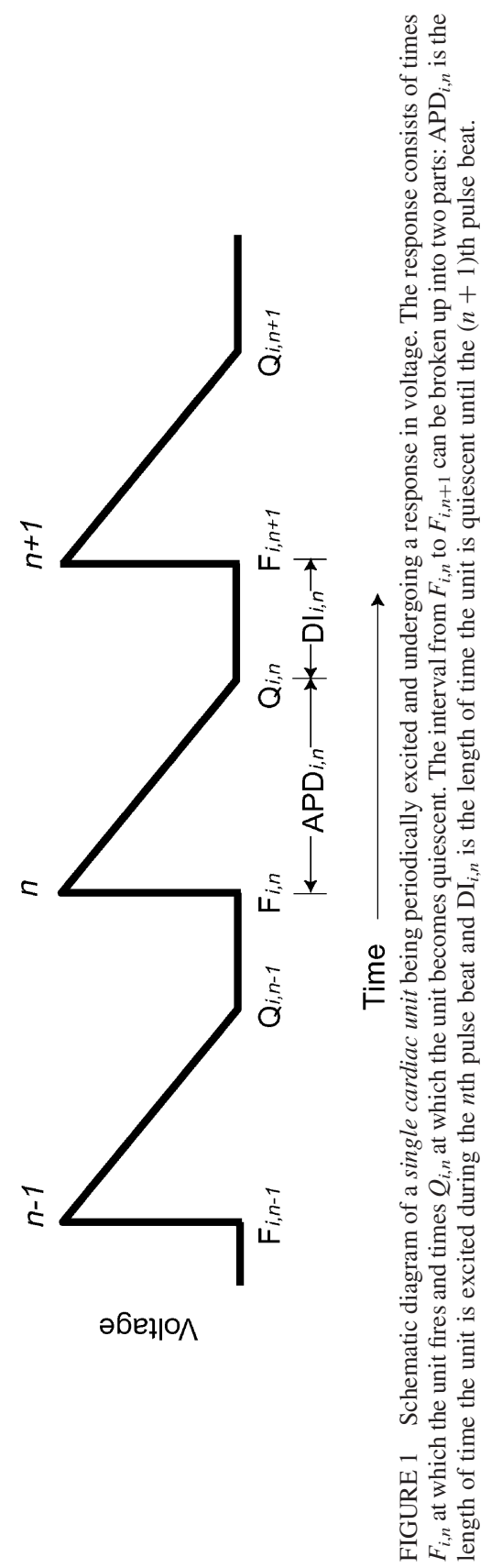


Note that $P_{i, n}$ is not identical to $P_{i+1, n}$ because the CV is not the same for the $n$th pulse beat as the $(n+1)$ th pulse beat. To resolve how $P_{i, n}$ is related to $P_{i+1, n}$ we observe (as shown in Fig. 2) that the leading edge of the $n$th wave of excitation propagates at speed $\mathrm{CV}_{i, n}$, so the front of wave $n$ reaches the $(i+1)$ th unit after a delay of $\Delta x / \mathrm{CV}_{i, n} . \Delta x$ is the cardiac unit spacing and is assumed to be constant throughout the chain. Similarly, the back of wave $n$ of excitation (which is the front of wave $n+1$ ) reaches the $(i+1)$ th unit after a delay of $\Delta x / \mathrm{CV}_{i, n+1}$. Thus, the change in the period from one cell to the next for a particular wave $n$ is given by

$$
P_{i+1, n}=P_{i, n}+\left(\frac{\Delta x}{\mathrm{CV}_{i, n+1}}-\frac{\Delta x}{\mathrm{CV}_{i, n}}\right)
$$

This is the fundamental governing equation. In this equation, we substitute Eq. (3) to give

$$
\mathrm{APD}_{i, n+1}+\mathrm{DI}_{i, n+1}+\frac{\Delta x}{\mathrm{CV}_{i, n+1}}=\frac{\Delta x}{\mathrm{CV}_{i, n}}+\mathrm{APD}_{i+1, n+1}+\mathrm{DI}_{i+1, n+1}
$$

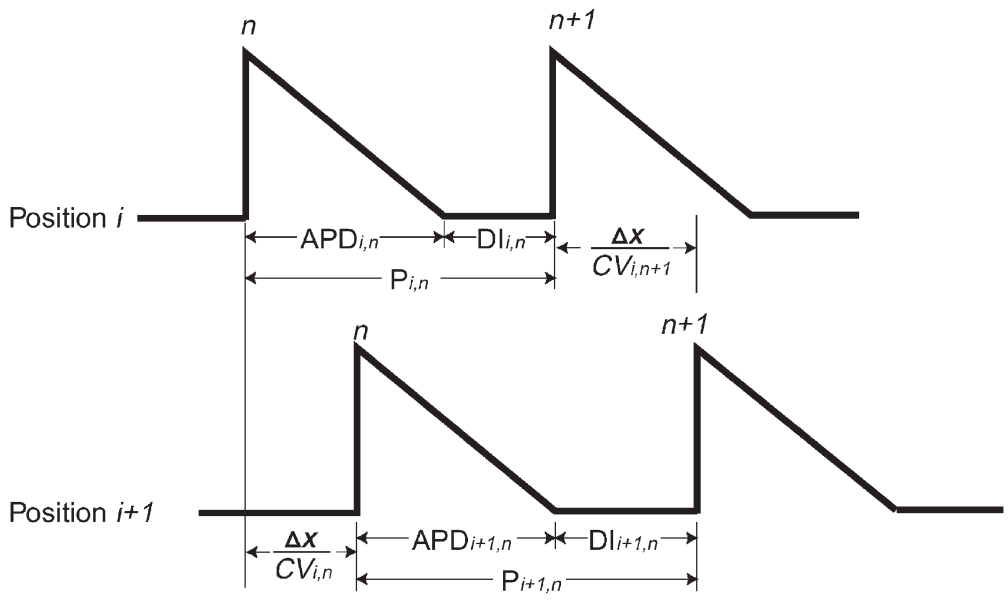

Time

FIGURE 2 Illustration of the derivation of the governing equation; relating the period $P_{i, n}$ of a pulse beat at spatial position $i$ to the period $P_{i+1, n}$ of the same pulse beat at spatial position $i+1$. 
Then, substituting Eqs. (1) and (2), defining $h(x)=\Delta x / g(x)$ and rewriting $u_{i, n} \equiv \mathrm{DI}_{i, n}$ for clarity, results in

$$
u_{i+1, n+1}+f\left(u_{i+1, n}\right)-u_{i, n+1}-f\left(u_{i, n}\right)+h\left(u_{i, n}\right)-h\left(u_{i, n+1}\right)=0
$$

which is a partial difference equation on the dependent variable $u_{i, n}$ with the independent variables $i, n$ which indicate space and time, respectively.

Although working with functions for $f$ and $h$ fitted directly from experimental data is possible (see Fig. 3), we further simplify the problem by taking $f$ and $h$ as idealized functions which give similar qualitative behavior to the experimentally determined functions, namely

$$
\begin{gathered}
f\left(u_{i, n}\right)=-1+\mu\left(1-u_{i, n}\left(1-u_{i, n}\right)\right), \\
h\left(u_{i, n}\right)=\alpha u_{i, n}+\beta .
\end{gathered}
$$

Extensive numerical simulations have shown that these functions capture the essential details of the more complicated system. Notice that Eq. (7) is a variant on the well known Logistic equation, as might be expected from the bifurcation diagram in Fig. 4 which looks similar to the corresponding diagram for the Logistic equation. Thus, the governing Eq. (6) becomes

$$
\begin{aligned}
& u_{i+1, n+1}-\mu u_{i+1, n}\left(1-u_{i+1, n}\right)-u_{i, n+1}+\mu u_{i, n}\left(1-u_{i, n}\right) \\
& \quad+\alpha\left(u_{i, n}-u_{i, n+1}\right)=0 .
\end{aligned}
$$

\section{PROBLEM STATEMENT}

As derived above, the system of interest is a partial difference equation which models a chain of cardiac cell units on a one-dimensional domain

$$
\begin{aligned}
u_{i+1, n+1}-\mu u_{i+1, n}\left(1-u_{i+1, n}\right)= & u_{i, n+1}-\mu u_{i, n}\left(1-u_{i, n}\right) \\
& +\alpha\left(u_{i, n+1}-u_{i, n}\right) .
\end{aligned}
$$



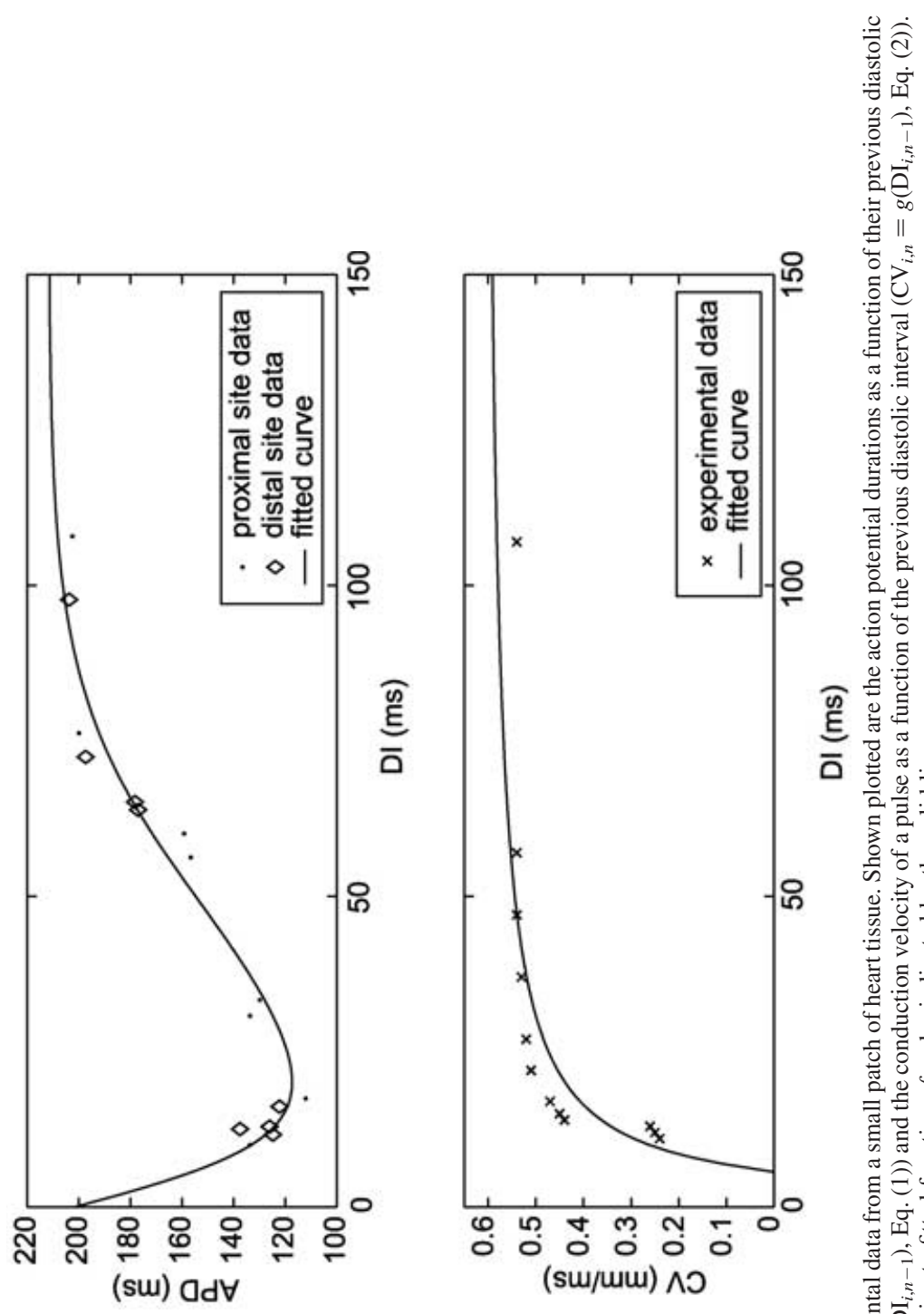

둥 冚焉

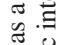

ติ

晃奇

콩

祃

ำ

.气 ก ฐ

ह

万

늘 믈

हैं

的范

ง.

药

$\because \frac{0}{\square}$

픙 으을

능

웡 흥

음

클으

施

$\exists \bigodot \Xi$

ษ

完宅

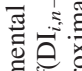

늘

这

a

$m \varangle$

뜬 을

它导造 


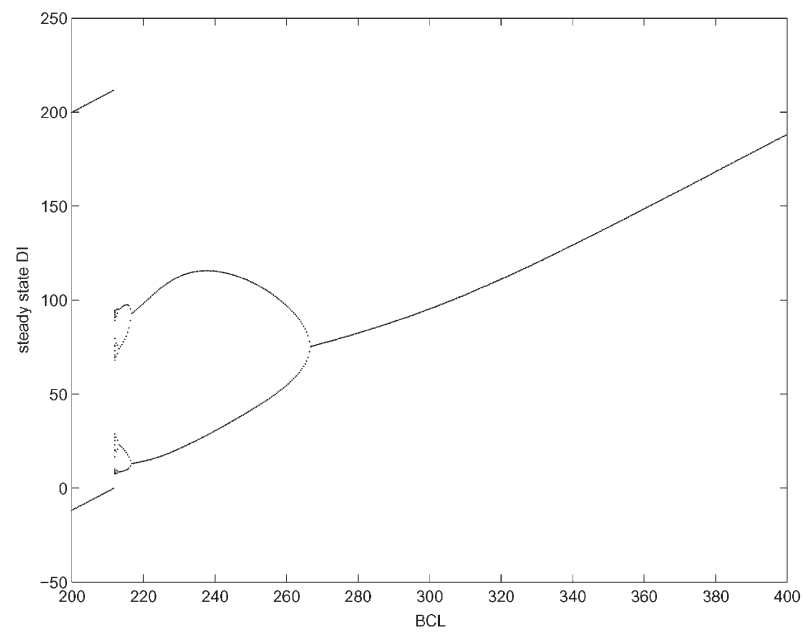

FIGURE 4 Bifurcation diagram for the single periodically excited cardiac unit. This diagram was generated numerically using the function $f$ shown in Fig. 3.

Here $i$ is the discrete space index, $n$ is the discrete time index, $u_{i, n}$ is the amplitude of the unit at a point $i$ in space at time $n$. Also, $\mu$ and $\alpha$ are parameters. The boundary condition at $i=0$ is taken as

$$
u_{0, n+1}=1+\mu u_{0, n}\left(1-u_{0, n}\right),
$$

that is, the unit at $i=0$ is driving the rest of the chain. Physically this corresponds to periodic stimulation of one end of the tissue by the experimenter. We will be interested in values of $\mu$ for which the unit at $i=0$ (a modified Logistic equation) is undergoing period-2 behavior. The boundary condition at $i=L$ (the other end of the chain) is not important because the coupling is "uni-directional", i.e. the oscillator at $i$ is only affected by the oscillator at $i-1$ and not the oscillator at $i+1$.

Being discrete in both indices makes simulation of the system on a computer particularly easy. Such simulations show that for a range of parameters $\mu$ and $\alpha$, a stable steady state is reached where each oscillator is undergoing period- 2 behavior in time, and for fixed $n$ has a complicated spatial structure. Two sequential snapshots of the chain at steady state are 
shown in Fig. 5. As time $n$ is incremented further, the pictures would just repeat, since the overall behavior is period- 2 in time.

The aim of our work on this problem is to understand the dynamics of the system as shown in Fig. 5 through the use of perturbation techniques.

\section{ANALYSIS}

To begin with, we introduce a small parameter $\epsilon$ into the problem by assuming that $\alpha$ in Eq. (10) is small, and we replace $\alpha$ by $\epsilon \alpha$ :

$$
\begin{aligned}
& u_{i+1, n+1}-\mu u_{i+1, n}\left(1-u_{i+1, n}\right)-u_{i, n+1}+\mu u_{i, n}\left(1-u_{i, n}\right) \\
& \quad+\epsilon \alpha\left(u_{i, n}-u_{i, n+1}\right)=0 .
\end{aligned}
$$
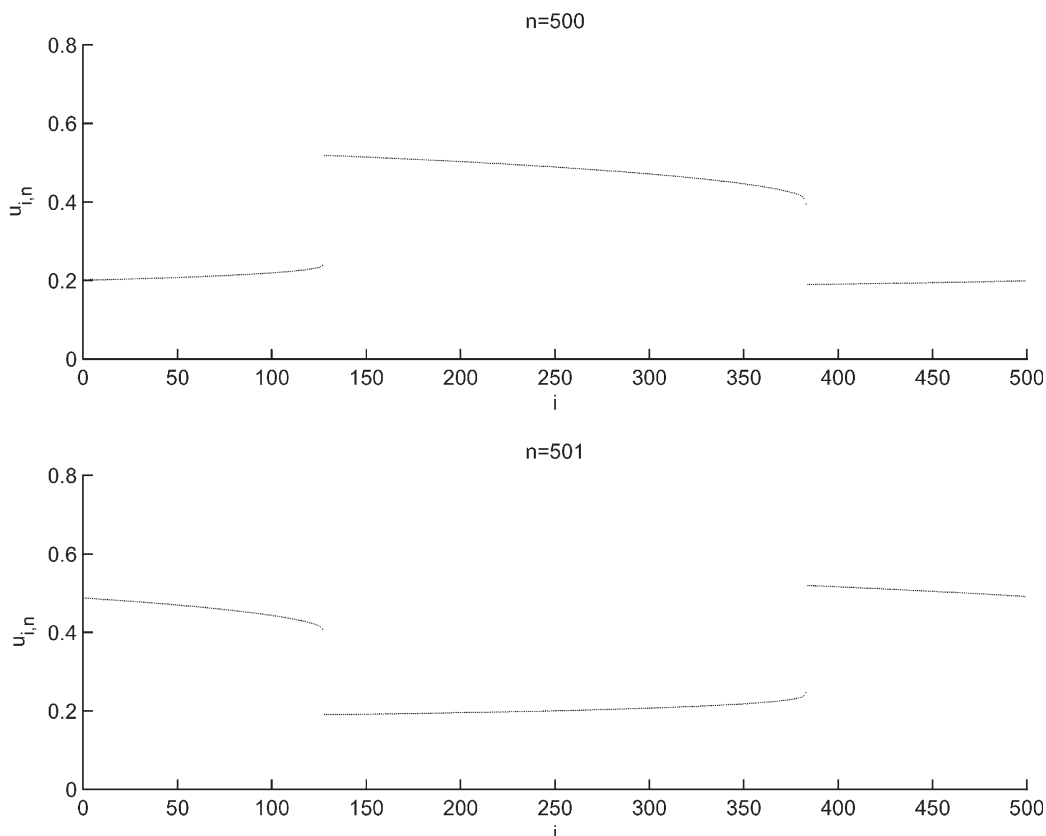

FIGURE 5 Sequential snapshots of the simulated partial difference equation (10) at steady state. Parameters used are $\mu=-3.2, \alpha=-.0003$. 


\section{Regular Perturbations}

Attempting to solve for the steady state with a regular perturbation expansion proceeds as follows. An ansatz is assumed of the form

$$
u_{i, n}=u_{i, n}^{0}+\epsilon u_{i, n}^{1}+O\left(\epsilon^{2}\right) .
$$

Plugging Eq. (13) into Eq. (12) and collecting terms gives

$$
\begin{gathered}
O\left(\epsilon^{0}\right): u_{i+1, n+1}^{0}-\mu u_{i+1, n}^{0}\left(1-u_{i+1, n}^{0}\right) \\
-u_{i, n+1}^{0}+\mu u_{i, n}^{0}\left(1-u_{i, n}^{0}\right)=0, \\
O\left(\epsilon^{1}\right): \quad u_{i+1, n+1}^{1}-\mu u_{i+1, n}^{1}\left(1-2 u_{i+1, n}^{0}\right)-u_{i, n+1}^{1} \\
+\mu u_{i, n}^{1}\left(1-2 u_{i, n}^{0}\right)+\alpha\left(u_{i, n}^{0}-u_{i, n+1}^{0}\right)=0,
\end{gathered}
$$

and the equations are solved sequentially. Equation (14) may be rewritten in a more transparent way, namely

$$
u_{i+1, n+1}^{0}-\mu u_{i+1, n}^{0}\left(1-u_{i+1, n}^{0}\right)=u_{i, n+1}^{0}-\mu u_{i, n}^{0}\left(1-u_{i, n}^{0}\right) .
$$

By observing that the LHS of the equation contains only terms with subscripts of $i+1$ and the RHS contains terms with subscripts of $i$, it may be concluded that

$$
u_{i, n+1}^{0}-\mu u_{i, n}^{0}\left(1-u_{i, n}^{0}\right)=C_{n}
$$

where $C_{n}$ is an arbitrary function of $n$ which is determined by the boundary condition. Applying Eq. (11) implies that $C_{n}=1$ for all $n$, so that

$$
u_{i, n+1}^{0}=1+\mu u_{i, n}^{0}\left(1-u_{i, n}^{0}\right) .
$$

Since we are interested in values of $\mu$ for which this equation exhibits period2 dynamics (as mentioned above), we write the solution to Eq. (18) as

$$
u_{i, n}^{0}=v_{1}+v_{2}(-1)^{n} .
$$

The perturbation method allows us to approximate steady state solutions which are periodic in time, but it cannot be used to approximate transient solutions. In Eq. (19), $v_{1}, v_{2}$ are constants which are determined completely by the boundary condition in Eq. (11). Specifically, $v_{1}=(\mu+1) / 2 \mu$, 
$v_{2}=\left(\sqrt{\mu^{2}+2 \mu-3}\right) / 2 \mu$. Carrying out the method to next order requires plugging Eq. (19) back into Eq. (15) and results in

$$
\begin{aligned}
& u_{i+1, n+1}^{1}+\left(-\mu+2 \mu v_{1}+2 \mu v_{2}(-1)^{n}\right) u_{i+1, n}^{1}-u_{i, n+1}^{1} \\
& -\left(-\mu+2 \mu v_{1}+2 \mu v_{2}(-1)^{n}\right) u_{i, n}^{1}+2 \alpha v_{2}(-1)^{n}=0 .
\end{aligned}
$$

This equation is a linear non-homogeneous partial difference equation with periodic coefficients. It may be rewritten more simply (see, for example, Ref. [6]) by using the shift-operator notation: $E_{1} u_{i, n}=u_{i+1, n}, E_{2} u_{i, n}=$ $u_{i, n+1}$ as

$$
\begin{array}{r}
E_{1} E_{2} u_{i, n}^{1}+\left(-\mu+2 \mu v_{1}+2 \mu v_{2}(-1)^{n}\right) E_{1} u_{i, n}^{1}-E_{2} u_{i, n}^{1} \\
-\left(-\mu+2 \mu v_{1}+2 \mu v_{2}(-1)^{n}\right) u_{i, n}^{1}+2 \alpha v_{2}(-1)^{n}=0
\end{array}
$$

which may be further factored to give

$$
\left[\left(E_{1}-1\right)\left(E_{2}-\mu+2 \mu v_{1}+2 \mu v_{2}(-1)^{n}\right)\right] u_{i, n}^{1}=-2 \alpha v_{2}(-1)^{n} .
$$

The homogeneous solution to Eq. (22) can be found by setting the LHS $=0$, and in general will involve two arbitrary functions of $i, n$ since the problem is of second order. The homogeneous solution is found to be

$$
\left(u_{i, n}^{1}\right)_{\text {homog }}=\left[\left(2 \mu v_{1}\right)^{2}-\left(2 \mu v_{2}\right)^{2}\right]^{n / 2} \phi_{i}+\psi_{n}, \quad \text { for } n=0,2,4, \ldots
$$

which contains two arbitrary functions $\phi_{i}, \psi_{n}$. The particular solution to Eq. (22) can be found immediately by an ansatz of the form $\left(u_{i, n}^{1}\right)_{\text {part }}=$ $i\left(a+b(-1)^{n}\right)$, and solving for $a, b$ such that Eq. (22) (now including the RHS) is satisfied. Doing so, we find that

$$
\left(u_{i, n}^{1}\right)_{\mathrm{part}}=i\left[\frac{-4 \alpha \mu v_{2}^{2}+\left(4 \mu v_{1}-2 \mu+2\right) \alpha v_{2}(-1)^{n}}{4 \mu^{2}\left(v_{2}^{2}-v_{1}^{2}\right)+4 \mu^{2} v_{1}-\mu^{2}+1}\right] .
$$

So, the total solution to Eq. (22) is

$$
\begin{aligned}
u_{i, n}^{1}= & {\left[\left(2 \mu v_{1}\right)^{2}-\left(2 \mu v_{2}\right)^{2}\right]^{n / 2} \phi_{i} } \\
& +\psi_{n}+i\left[\frac{-4 \alpha \mu v_{2}^{2}+\left(4 \mu v_{1}-2 \mu+2\right) \alpha v_{2}(-1)^{n}}{4 \mu^{2}\left(v_{2}^{2}-v_{1}^{2}\right)+4 \mu^{2} v_{1}-\mu^{2}+1}\right], \\
& \quad \text { for } n=0,2,4, \ldots
\end{aligned}
$$


The main result from all this analysis is to show that the solution is secular in space since it contains terms proportional to $i$, and hence the regular perturbation approximation is only valid for $i \sim O(1)$.

\section{Singular Perturbations}

The secular terms in Eq. (25) may be eliminated by using the method of multiple scales (see, for example, Ref. [5]). The multiple scales procedure consists of assuming the existence of two space scales: regular space $i$ and "stretched" space $s \equiv \epsilon i$. The method assumes that $u$ depends explicitly on $i, s$ and $n$. Expanding $u_{i, n}$ of Eq. (12) in a perturbation series, we get

$$
u_{i, n}=u_{i, s, n}^{0}+\epsilon u_{i, s, n}^{1}+O\left(\epsilon^{2}\right) .
$$

Note that as $i \rightarrow i+1, s=\epsilon i \rightarrow \epsilon(i+1)=s+\epsilon$. Consequently, shifting the index $i \rightarrow i+1$ in Eq. (26) produces

$$
u_{i+1, n}=u_{i+1, s+\epsilon, n}^{0}+\epsilon u_{i+1, s+\epsilon, n}^{1}+O\left(\epsilon^{2}\right) .
$$

Expanding Eq. (27) in powers of $\epsilon$ gives

$$
u_{i+1, n}=u_{i+1, s, n}^{0}+\epsilon\left(u_{i+1, s, n}^{1}+\frac{\partial u_{i+1, s, n}^{0}}{\partial s}\right)+O\left(\epsilon^{2}\right)
$$

Equations (26) and (28) are the new perturbation ansatzes, to be substituted into Eq. (12).

Since the method is identical to regular perturbations at $O\left(\epsilon^{0}\right)$, the solution to $u_{i, s, n}^{0}$ is still given by Eq. (19), i.e.

$$
u_{i, s, n}^{0}=v_{1}+v_{2}(-1)^{n}
$$

Now, however, $v_{1}, v_{2}$ (which were constants) are now allowed to be explicit functions of the stretched space variable $s$ :

$$
u_{i, s, n}^{0}=v_{1}(s)+v_{2}(s)(-1)^{n}
$$


This equation, along with Eq. (28) is plugged back into Eq. (12) and terms of $O\left(\epsilon^{1}\right)$ are collected to give

$$
\begin{aligned}
u_{i+1, s, n+1}^{1}-u_{i, s, n+1}^{1}+\left(2 \mu v_{2}(-1)^{n}+2 \mu v_{1}-\mu\right) u_{i+1, s, n}^{1} \\
+\left(-2 \mu v_{2}(-1)^{n}-2 \mu v_{1}+\mu\right) u_{i, s, n}^{1} \\
=\left[-2 \mu v_{1} v_{2}^{\prime}-2 \mu v_{1}^{\prime} v_{2}+(\mu+1) v_{2}^{\prime}-2 \alpha v_{2}\right](-1)^{n} \\
\quad-2 \mu v_{1} v_{1}^{\prime}-2 \mu v_{2} v_{2}^{\prime}+(\mu-1) v_{1}^{\prime}
\end{aligned}
$$

where ()$^{\prime} \equiv \mathrm{d}() / \mathrm{d} s$. Here we need only to remove resonant terms in the particular solution, so we ignore the homogeneous solution and concentrate on the RHS of Eq. (31). Two groups of troublesome terms appear there: those which are not explicit functions of $i$ and $n$, and those which are not explicit functions of $i$ but depend on $n$ in the functional form of $(-1)^{n}$. Non-zero terms from either group will result, as we saw above, in secular growth proportional to $i$. Each group of terms must be set to zero independently of the other, because $v_{1}, v_{2}$ are not allowed to be functions of either $i$ or $n$. Doing so, we arrive at two coupled "slow-flow" equations for the two slow variables $v_{1}(s), v_{2}(s)$ :

$$
\begin{gathered}
-2 \mu v_{1} v_{2}^{\prime}-2 \mu v_{1}^{\prime} v_{2}+(\mu+1) v_{2}^{\prime}-2 \alpha v_{2}=0, \\
-2 \mu v_{1} v_{1}^{\prime}-2 \mu v_{2} v_{2}^{\prime}+(\mu-1) v_{1}^{\prime}=0,
\end{gathered}
$$

where primes denote differentiation with respect to $s$. The second of these equations may be integrated directly to give

$$
-\mu v_{1}^{2}-\mu v_{2}^{2}+(\mu-1) v_{1}=H
$$

The constant $H$ may be determined by enforcing the boundary condition at $s=0$, i.e. the left-hand end of the chain. Consequently, using the boundary condition $v_{1}(s=0)=(\mu+1) / 2 \mu, v_{2}(s=0)=\left(\sqrt{\mu^{2}+2 \mu-3}\right) / 2 \mu$ in Eq. (34) gives that $H=-1$. Thus, the first integral may be rewritten as

$$
v_{2}= \pm \sqrt{\frac{-\mu v_{1}^{2}+(\mu-1) v_{1}+1}{\mu}}
$$


Substituting this into Eq. (32) gives a single first order ODE

$$
\frac{\mathrm{d} v_{1}}{\mathrm{~d} s}=-\frac{4 \alpha\left(v_{1}-1\right)\left(\mu v_{1}+1\right)}{8 \mu^{2} v_{1}^{2}-8 \mu^{2} v_{1}+4 \mu v_{1}+\mu^{2}-4 \mu-1} .
$$

with the boundary condition $v_{1}(s=0)=(\mu+1) / 2 \mu$. Although this equation can be integrated in closed form giving an analytical expression for $s$ in terms of $v_{1}$, for brevity we numerically integrate Eq. (36) to obtain $v_{1}(s)$ and then use Eq. (35) to obtain $v_{2}(s)$. We substitute these results into Eq. (30) and plot in Fig. 6 the resulting values of $u_{i, s, n}^{0}$ together with the results of numerical simulation of Eq. (10) that was shown in Fig. 5. Although the agreement appears to be excellent, the perturbation solution ceases to exist at the point where the denominator of Eq. (36) vanishes. We deal with this problem in "Singularities" section of the paper.

The slow-flow equations given in Eqs. (32) and (33) can also be used to quantitatively answer various questions about the system. For example, it is possible to derive an exact (to the order of the perturbation method)

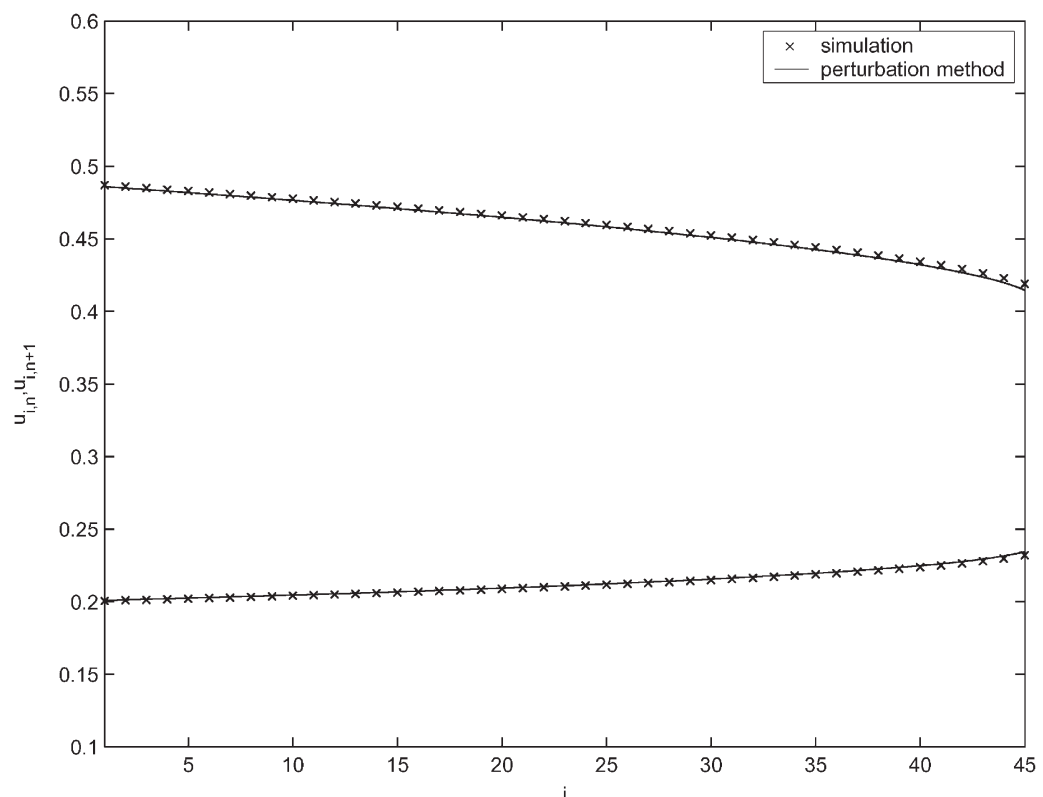

FIGURE 6 Comparison of numerical simulation and singular perturbation method Parameters used are $\mu=-3.2, \alpha=-0.001$. 
expression for where in space $(i)$ the first singularity occurs in the chain (c.f. Fig. 5). Using the analytical solution to Eq. (36) and substituting in the value of $v_{1}$ for which the denominator of Eq. (36) equals zero, the desired result for the spatial position $i^{\star}$ of the first singularity is obtained:

$$
\begin{aligned}
i^{\star}= & -\frac{1}{4 \alpha \epsilon}\left\{(\mu+3) \log \left(\sqrt{2 \mu^{2}+4 \mu+3}+2 \mu+3\right)\right. \\
& +(1-\mu) \log \left(\sqrt{2 \mu^{2}+4 \mu+3}-2 \mu-1\right) \\
& \times(-\mu-3) \log (\mu+3)-2 \sqrt{2 \mu^{2}+4 \mu+3} \\
& +\log (1-\mu) \mu-\log (1-\mu)-4 \log 2+6\}
\end{aligned}
$$

For $\mu=-3.2, \epsilon \alpha=-0.0003$, Eq. (37) gives $i^{\star}=152.0$ which agrees approximately with the results of direct simulation of the original Eq. (12), c.f. Fig. 5.

\section{SINGULARITIES}

The singular perturbation method used above matches the numerical solution of the chain quite well up to a point: the ODE's in Eqs. (32) and (33) have a singularity, where the derivatives become infinite. This problem arises because the ansatz used in Eq. (28) tacitly assumes that the solution we are interested in can be approximated by a continuous function. Continuity is assumed because we assumed the derivative $\left(\partial u_{i+1, s, n}^{0}\right) / \partial s$ exists. Of course, since we are dealing with a discrete governing equation, the actual solution is never continuous; we would expect the method to work if the solution is slowly varying enough and has no large jumps. From examining Fig. 5, it is evident that there do exist places in space where the solution undergoes large discrete jumps in value. We have found that the singular perturbation method cannot replicate this discontinuity; the ODE's in Eqs. (32) and (33) have a singularity at this point. This section of the paper deals with how we chose to resolve this issue.

To finish the approximation and find out what happens after the singularity, we return to the original partial difference equation Eq. (10) and use the following reasoning. We assume that at a particular point in space 
(denoted $i^{-}$), a single cell is existing exactly at the values predicted by the singularity in the slow flow. We denote by $u_{n}^{-}$the values that this cell takes on. Our goal at this point is to determine the behavior of the cardiac unit at $i^{+}\left(=i^{-}+1\right)$, i.e. immediately to the right of the singularity.

The cardiac unit at the location $i^{-}$by hypothesis is undergoing behavior which is represented by

$$
u_{n}^{-}=v_{1}^{-}+v_{2}^{-}(-1)^{n}
$$

where $v_{1,2}^{-}$are the values where the denominators of Eqs. (32) and (33) vanish, specifically

$$
\begin{aligned}
& v_{1}^{-}=\frac{\sqrt{2 \mu^{2}+4 \mu+3}+2 \mu-1}{4 \mu}, \\
& v_{2}^{-}=\frac{\sqrt{-\sqrt{2 \mu^{2}+4 \mu+3}+\mu^{2}+2 \mu}}{2 \sqrt{2} \mu}
\end{aligned}
$$

Using $u_{n}^{-}, u_{n+1}^{-}$for $u_{i, n}, u_{i, n+1}$ in Eq. (10), using Eq. (38), and denoting $u_{i+1, n}$ as $u_{n}^{+}$, we have

$$
\begin{aligned}
u_{n+1}^{+}= & \mu u_{n}^{+}\left(1-u_{n}^{+}\right)+\left[v_{1}^{-}\left(-\mu+1+\mu v_{1}^{-}\right)+\mu\left(v_{2}^{-}\right)^{2}\right] \\
& +\left[2 \mu v_{1}^{-}-\mu-2 \alpha-1\right] v_{2}^{-}(-1)^{n} .
\end{aligned}
$$

which is just an ordinary difference equation in $n$ on $u_{n}^{+}$. By simulating this equation for given parameters we can obtain information about the solution that is missing from the slow-flow equations in the previous section.

Samples of the time history of Eq. (40) are shown in Figs. 7 and 8 for $\mu=-3.2$ and $\mu=-3.35$, respectively. The initial condition in both cases is $u_{0}^{+}=v_{1}^{-}+v_{2}^{-}$. From these figures, we see that as $n$ (time) increases, the amplitude "switches phase". That is, at steady state, $u_{n}^{+}$ends up being out of phase with $u_{n}^{-}$. This phase-switching phenomenon explains the jumps in phase which appear in Fig. 5.

The steady-state amplitude of $u_{n}^{+}$as displayed in Figs. 7 and 8 can be characterized by the bifurcation diagram which is shown in Fig. 9. It is interesting that this bifurcation diagram (which is only over values of $\mu$ for which the first cell in the chain exhibits period-2 behavior) shows that the next cell after the singularity may exhibit period-2, period-4, 


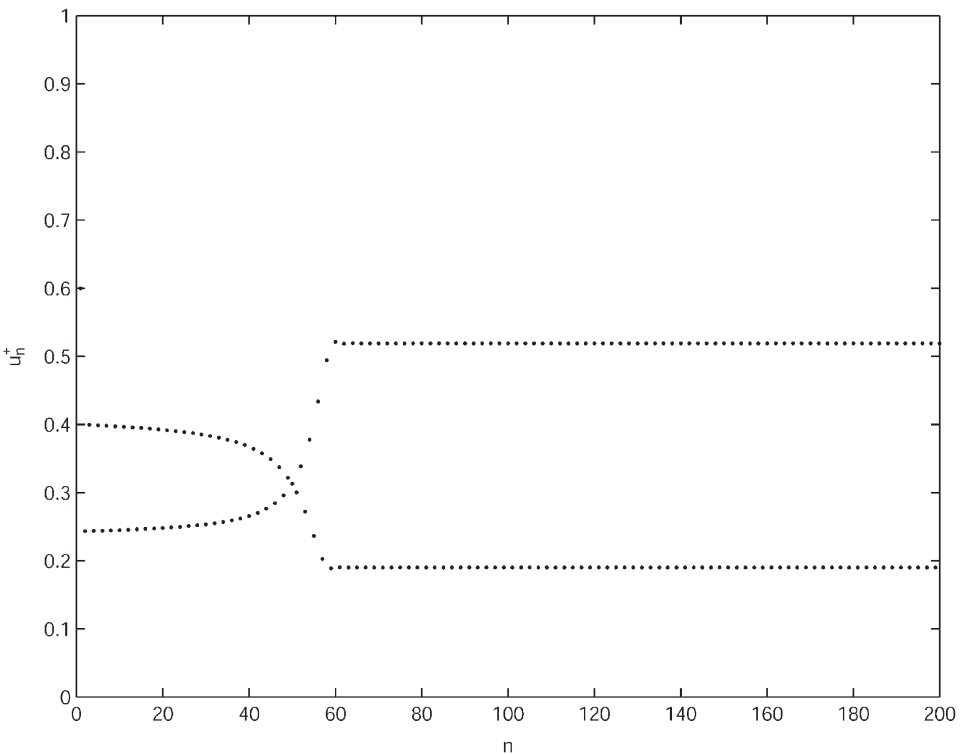

FIGURE 7 Time history of a simulation of Eq. (40). $\mu=-3.2$.

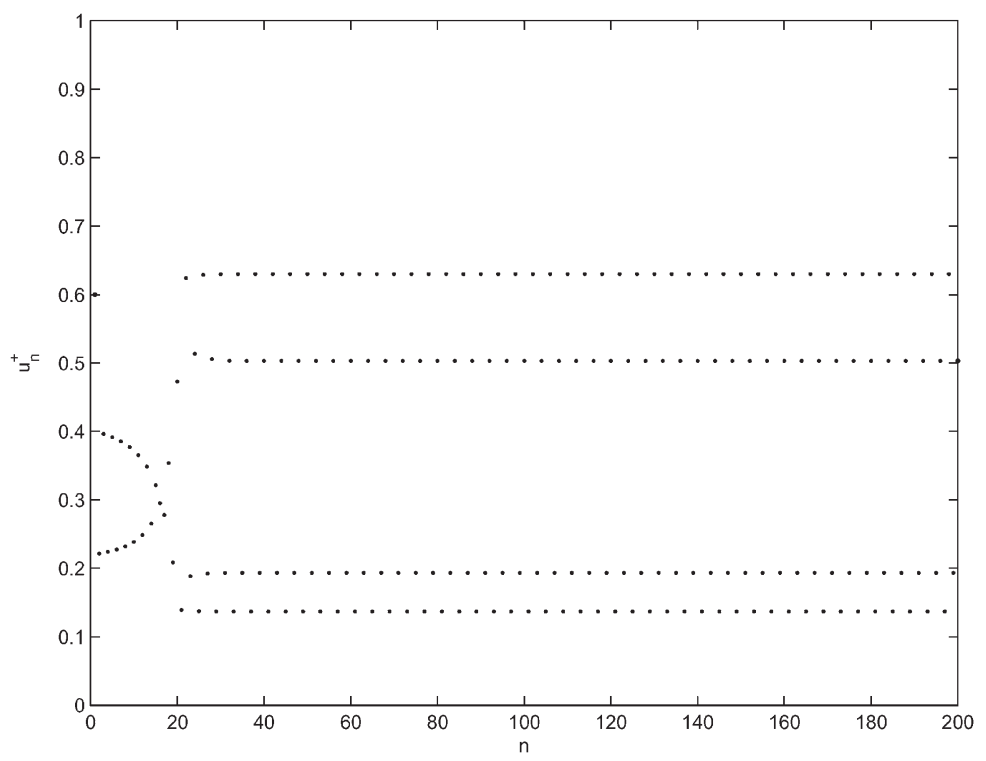

FIGURE 8 Time history of a simulation of Eq. (40). $\mu=-3.35$. 


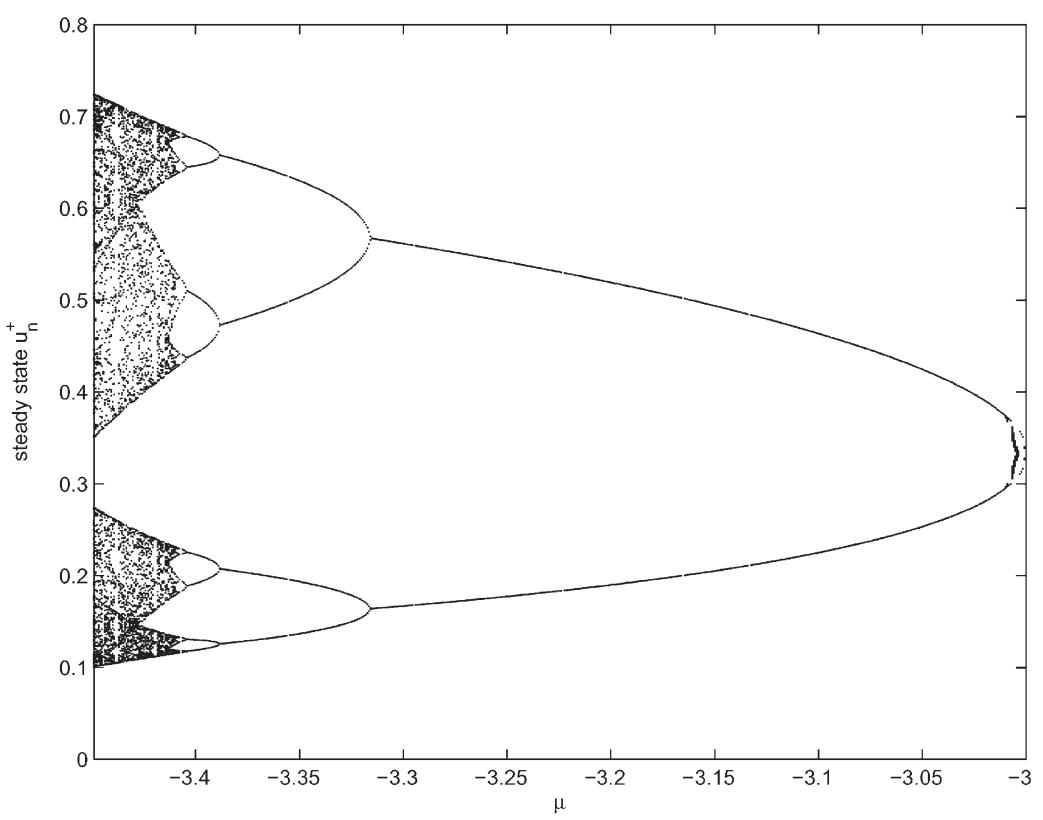

FIGURE 9 Numerically produced bifurcation diagram for the steady state solutions of Eq. (40) as a function of the bifurcation parameter $\mu$. Note that this diagram (which is only over values of $\mu$ for which the first cell in the chain exhibits period-2 behavior) indicates that the first cell after the singularity may be undergoing higher-periodic or even chaotic behavior.

period-8, etc. behavior (including chaotic behavior) depending on the value of $\mu$. This corresponds to the prediction of the appearance of higher-periodic or chaotic behavior at steady state in the chain of cardiac units while the first cell is being forced into period-2 motion, an effect we refer to as a "spatial bifurcation".

To see that this actually happens in the model system of Eq. (10), we present in Fig. 10 simulations of the chain for various values of $\mu$. In Fig. 10(a), which is a repeat of Fig. 5, all parts of the chain are undergoing period-2 behavior, which is apparent from the two values that each point in space takes on as time $n$ is increased. In Fig. 10(b), however, immediately after the singularity, a small part of the chain is undergoing period-4 behavior (indicated by the four different values that part of the chain takes on). This is expected from our analysis because for that particular value of $\mu=-3.35$, the bifurcation diagram in Fig. 9 predicts that the cell immediately to the right of the singularity will undergo period-4 behavior. 
The remaining quadrants of Fig. 10 are understood in the same way. So, in summary, although the outer solution breaks down at a singularity in the slow flow, by referring to Fig. 9, one can predict the extent of the spatial bifurcation in the chain.

The final part of our analysis is aimed at understanding the transition from the higher-period behavior back to period-2 behavior which is observed as we move along the chain by increasing location $i$. See, e.g. Fig. 10(b) in which period-4 behavior transitions into period-2 behavior further along the chain. It turns out that this evolution is well approximated by another outer-type solution. Specifically, for the case shown in Fig. 10(b), one assumes that the first cell is undergoing period-4 behavior
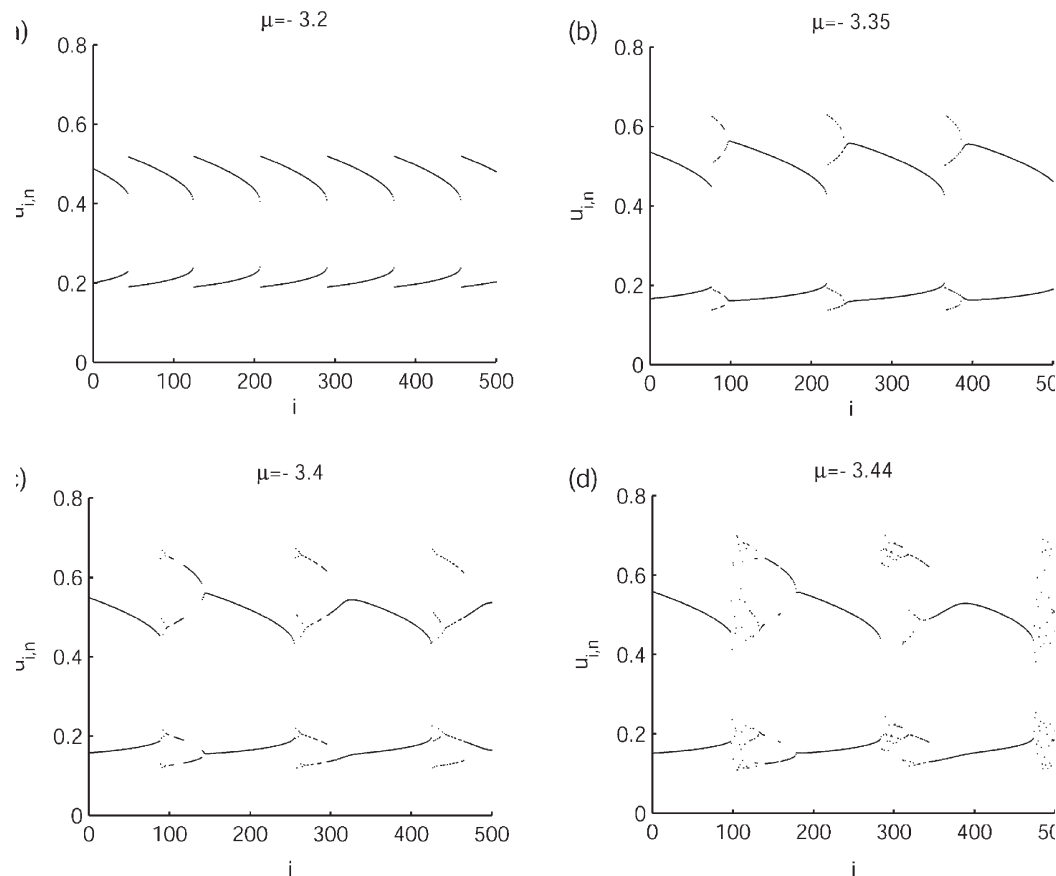

FIGURE 10 Shown in each quadrant are two sequential snapshots of the chain for a particular value of the parameter $\mu$ ( $\alpha=-0.001$ in all quadrants). In (a), each part of the chain is undergoing period- 2 behavior in time (hence the two dots for each value of $i$ ), however, the amplitude of the motion varies along the length. As $\mu$ is decreased further, parts of the chain bifurcate to period- 4 behavior, then period- 8 behavior, until (d), where a whole range of motions can be found occurring simultaneously at different parts of the chain, from periodic to chaotic. 
and modifies the singular perturbation method appropriately. First, the new perturbation ansatz (c.f. Eq. (30)) is taken to be

$$
u_{i, s, n}^{0}=v_{1}(s)+v_{2}(s) \cos (n \pi)+v_{3}(s) \sin (n \pi / 2)+v_{4}(s) \cos (n \pi / 2) .
$$

This form is chosen because it is possible to represent any period-4 motion by choosing appropriate values of $v_{1,2,3,4}$. The rest of the perturbation scheme is carried out exactly as above, with the exception of when it comes to eliminating secular terms. The coefficients of the constant term, and of the $\cos (n \pi), \sin (n \pi / 2)$, and $\cos (n \pi / 2)$ terms must all be set to zero, thus producing four coupled ODE's. For brevity, these are not reprinted here, but their numerical solution for $\mu=-3.35$; $\alpha=$ -0.001 is shown in Fig. 11. As can be seen, the method captures the transition from period-4 to period-2 behavior quite well, up until the point where the next singularity is reached. In summary, by pasting together outer solutions, along with the information provided by the bifurcation diagram Fig. 9, the total dynamics of the chain can be understood.

\section{CONCLUSIONS}

We have demonstrated, though the use of numerical simulations and singular perturbation analysis, that the partial difference equation studied in this work, Eq. (10), exhibits a "spatial bifurcation" structure at steady state. That is, for certain parameter ranges, parts of the chain may be oscillating in period-2 motion while other parts may be oscillating in higher periodic motion or even chaotic motion. The spatial structure of the solution may be understood as regions of outer solutions (from ODE's) which are connected together at singularities where the ODE's break down. The behavior at the singularities is understood through the use of Fig. 9 which predicts the dynamics of the first cell to the right of the singularity for the given parameter value(s).

The spatial bifurcation phenomenon observed in the present model is due in part to a feature of the assumed form of the restitution function $f$ in Eq. (7), specifically that $f$ is non-monotonic. Many experiments have shown that real restitution functions can be non-monotonic, and hence the spatial bifurcation structure that we observe may play an important role in explaining complex cardiac rhythms that are observed experimentally. 


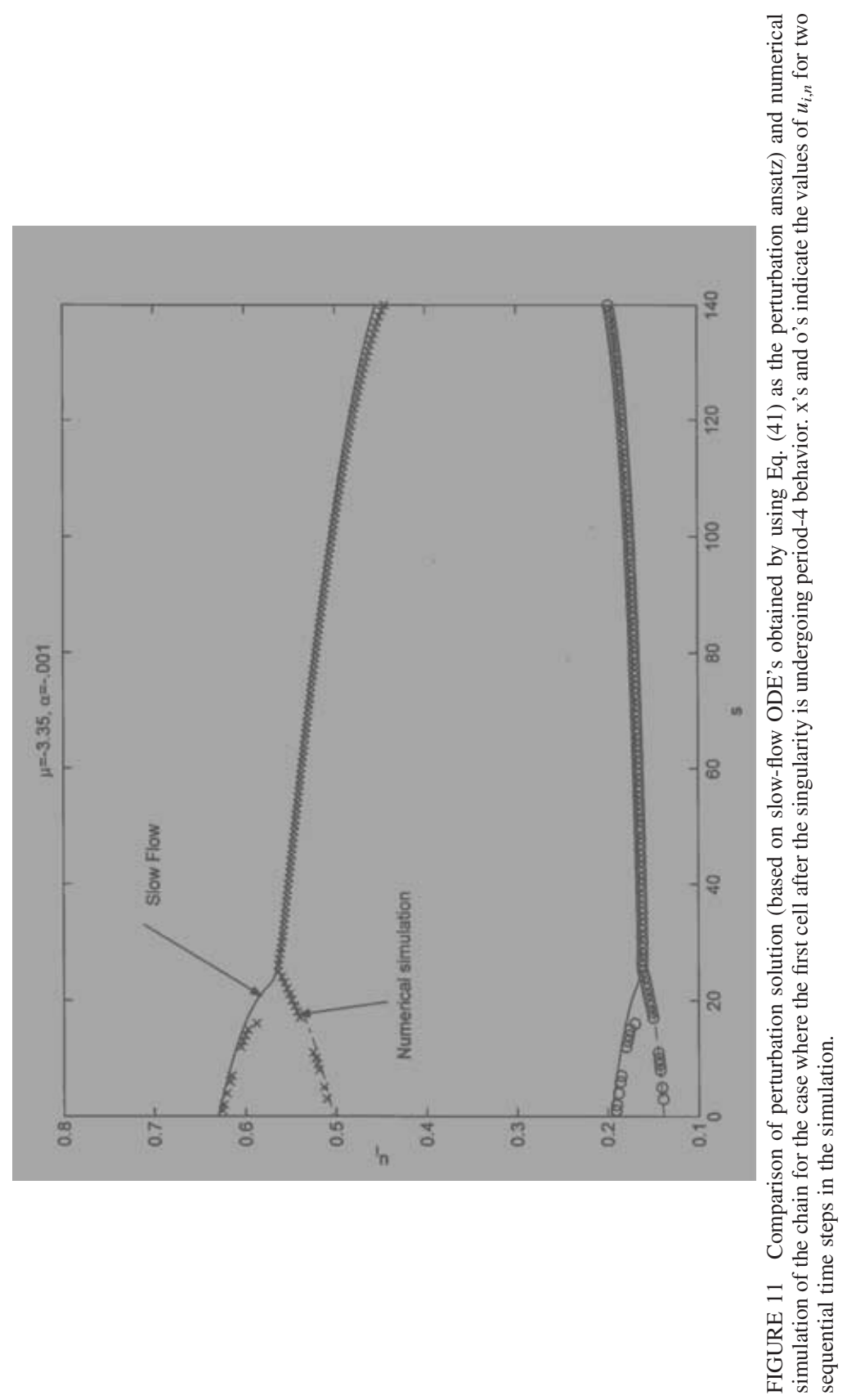


Extensions of this work could include the relaxation of certain simplifying assumptions to obtain a model which is more biologically realistic. This could include the inclusion of diffusion of current along the chain, and/or a more realistic choice for the dispersion function used in Eq. (8). See Refs. $[2,9,10]$ for recent approaches which include the effects of diffusion in models.

\section{References}

[1] D. R. Chialvo, R. F. Gilmour, Jr. and J. Jalife, Low dimensional chaos in cardiac tissue, Nature, 343 (1990), 653-657.

[2] B. Echebarria and A. Karma, Instability and spatiotemporal dynamics of alternans in paced cardiac tissue., arXiv: cond-mat/0111552 v1, 2001.

[3] R. F. Gilmour, M. Watanabe and N. Otani, Restitution properties and dynamics of reentry, Cardiac Electrophysiology: From Cell to Bedside, 3rd Ed., W. B. Saunders Company, London, pp 378-385, 1999.

[4] M. R. Guevara, G. Ward, A. Shrier and L. Glass, Electrical alternans and period-doubling bifurcations, Comput. Cardiol., (1984), 167-170.

[5] M. H. Holmes, Introduction to Perturbation Methods, Springer-Verlag, New York, 1995.

[6] R. E. Mickens, Difference Equations: Theory and Applications, Van Nostrand Reinhold, New York, 1990.

[7] R. J. Myerburg, K. M. Kessler, S. Kimura, A. L. Bassett, M. M. Cox and A. Castellanos, Life-threatening ventricular arrhythmias: the link between epidemiology and pathophysiology, Cardiac Electrophysiology: From Cell to Bedside, 2nd Ed., W. B. Saunders Company, London, pp 723-731, 1995.

[8] N. F. Otani and R. F. Gilmour, Memory models for the electrical properties of local cardiac systems, J. Theor. Biol., 187 (1997), 409-436.

[9] A. Vinet, Quasiperiodic circus movement in a loop model of cardiac tissue: Multistability and low dimensional equivalence, Ann. Biomed. Eng., 28 (2000), 704-720.

[10] M. A. Watanabe, F. H. Fenton, S. J. Evans, H. M. Hastings and A. Karma, Mechananism for discordant alternans, J. Cardiovasc. Electrophysiol., 12(2) (2001), 196-206. 